\title{
Copper sulfide nanoparticles as hole-transporting-material in a fully- inorganic blocking layers n-i-p perovskite solar cells: Application and working insights
}

\author{
Juan Tirado $^{\mathrm{a}}$, Cristina Roldán-Carmona ${ }^{\mathrm{b}, *}$, Fabio A. Muñoz-Guerreroc ${ }^{\mathrm{c}}$, Gemay Bonilla-Arboleda ${ }^{\mathrm{c}}$, \\ Maryline Ralaiarisoa $^{\mathrm{d}}$, Giulia Grancini ${ }^{\mathrm{b}}$, Valentin I.E. Queloz ${ }^{\mathrm{b}}$, Norbert Koch ${ }^{\mathrm{d}}$, \\ Mohammad Khaja Nazeeruddin ${ }^{\mathrm{b}, *}$, Franklin Jaramillo ${ }^{\mathrm{a}, *}$ \\ ${ }^{a}$ Centro de Investigación, Innovación y Desarrollo de Materiales CIDEMAT, Universidad de Antioquia UdeA, Calle 70 No. 52-21, Medellín, Colombia \\ ${ }^{\mathrm{b}}$ Group for Molecular Engineering of Functional Materials, Institute of Chemical Sciences and Engineering, Ecole Polytechnique Fédérale de Lausanne (EPFL), Sion, \\ Switzerland \\ ${ }^{\mathrm{c}}$ Research and Development Center, Andercol S.A., Carrera 64C No 95-84, Autopista Norte, Medellín, Colombia \\ ${ }^{\mathrm{d}}$ Institut für Physik, Humboldt-Universität zu Berlin, Brook-Taylor-Strasse 6, 12489 Berlin, Germany
}

\section{A R T I C L E I N F O}

\section{Keywords:}

Copper sulfide

Inorganic hole-transporting-material

Interfaces

Perovskite solar cells

\begin{abstract}
A B S T R A C T
One of the challenges in the field of perovskite solar cells (PSC) is the development of inorganic hole-transporting-materials (HTM) suitable for solution-processed deposition, in order to have cheaper, more stable and scalable devices. Herein, we report the synthesis and characterization of p-type copper sulfide nanoparticles for their application for the first time as a low-cost, fully-inorganic HTM in mesoscopic n-i-p PSC. By employing CuS combined with two different perovskites, $\mathrm{CH}_{3} \mathrm{NH}_{3} \mathrm{PbI}_{3}\left(\mathrm{MAPbI}_{3}\right)$ and $\left(\mathrm{FAPbI}_{3}\right)_{0.78}\left(\mathrm{MAPbBr}_{3}\right)_{0.14}\left(\mathrm{CsPbI}_{3}\right)_{0.08}$ (CsFAMAPbIBr), very high current densities and fill-factors are observed, suggesting an effective hole-extraction happening at the CuS interface. Noticeable, our cells exhibit one of the highest power conversion efficiencies (PCE) in n-i-p configuration employing a sole solution-processed inorganic HTM via non-toxic solvents, leading to $13.47 \%$ and $11.85 \%$ for $\mathrm{MAPbI}_{3}$ and $\mathrm{CsFAMAPbIBr}$, respectively. As a remark, such PCE values are only limited by a reduced open-circuit voltage around $0.8 \mathrm{~V}$, due to different phenomena occurring at perovkite/CuS interface such as an increased non-radiative recombination, caused by considerable difference in valence band value, and the effect of CuS metallic character. Overall, these findings highlight CuS as an extremely cheap alternative to common organic HTMs and pave the way to new improvements employing this material in fullinorganic blocking layers PSC.
\end{abstract}

\section{Introduction}

Since the first demonstration of solar devices comprising metal halide perovskites [1], perovskite solar cells (PSCs) have been the subject of an intense investigation in the photovoltaic field, yielding certified power conversion efficiencies (PCE) over 23\% at laboratory scale [2]. Their outstanding performance, comparable to polycrystalline silicon solar cells, together with their facile fabrication methodologies, convert these materials in a very promising alternative for low cost photovoltaics $[3,4]$. Nevertheless, to ensure an efficient hole and electron extraction, PSCs require the presence of additional charge-transport layers, which usually represent the major bottleneck towards low- cost commercialization [5]. In particular, the hole-transport materials (HTMs) generally employed in high efficient devices are mostly limited to octakis(4-methoxyphenyl)-9,9'-spirospirobi[9H-fluorene]- 2,2',7,7'tetramine (spiroOMeTAD) [6], few conjugated small molecules [7-9] and polymers $[10,11]$, which exhibit limited long-term stability and considerable materials cost [12-14]. In addition, due to their low hole mobility, organic HTMs usually require the incorporation of hygroscopic additives, typically lithium and/or cobalt salts, to enhance the charge carrier-transport properties. However, they also promote additional degradation in the photoactive material [15]. Hence, searching for an efficient, stable, and low-cost HTM has become vital to accomplish a future large-scale commercialization. 
In this context, inorganic HTMs represent a promising alternative due to the combination of low-cost, high hole mobility and conductivity, as well as their high chemical and thermal stability [16]. Despite few examples are present in the literature, which includes CuI [17], CuSCN [18], $\mathrm{NiO}$ [19], $\mathrm{Cu}_{2} \mathrm{O}$ [20], graphene oxide [21], carbon nanotubes (CNTs) [22], CIGSSe nanocrystals [23] or $\mathrm{FeS}_{2}$ [24], many of them require high temperature to properly crystalize, while those processed at low temperatures are often applied in combination with Spiro-OMeTAD $[25,26]$. Consequently, very few reports employ the sole inorganic material as HTM. In addition, these materials are regularly processed in polar solvents that can easily damage the perovskite layer, further limiting their application to the n-i-p device configuration.

Among the above restrictions, few interesting inorganic alternatives have appeared in literature. For example, recent investigation demonstrates a promising efficiency of $\sim 16 \%$ for a mesoscopic n-i-p configuration employing CuSCN [27] which further improves to $20.2 \%$ when including reduced graphene oxide [28]. Unfortunately this material requires the use of toxic and dangerous solvents such as diethyl sulfide, highly undesirable for scaling up and industrial processes. Similar efficiencies have been also obtained for CNTs composites when they are functionalized with poly (3-hexylthiophene), yet the insertion of such organic semiconductor increases considerably the materials cost [22]. As a consequence, it is mandatory to develop low-temperature, stable and efficient inorganic HTMs which are compatible with low-cost and large scale deposition methods.

In this framework, earth abundant and extremely cheap copper sulfide derivatives $\left(\mathrm{Cu}_{2-\mathrm{x}} \mathrm{S}, 0>\mathrm{x}>1\right)$ represent an interesting class of p-type semiconductors with tunable optical properties and high charge-transport mobility, conductivity and chemical stability $[29,30]$. Among the different stable phases with variable Cu:S ratio, covellite (CuS) presents the highest concentration of valence-band-delocalized holes, resulting in an unusual p-type metallic-like character with dual semiconductor-plasmonic nature [31]. Due to such electronic properties, CuS has been successfully employed for different optoelectronic applications, including the tuning of the ITO work function [32], or the passivation of the HTM/metal interface via CuS sublimation [25]. Moreover, CuS behaves as inorganic barrier against environmental moisture [25], which could prevent perovskite materials from degradation. However, despite all these benefits, CuS has not been yet employed as a sole inorganic HTM, mainly due to the lack of precursor solutions compatible with the perovskite material [33]. In addition, CuS synthesis normally require the use of high temperatures $\left(200-500{ }^{\circ} \mathrm{C}\right)$ $[34,35]$ or highly toxic materials, and due to its narrow thermodynamic stability domain, its selective growth can be more restrictive compared to other phases, hindering its fabrication [36].

In this article, we present the synthesis and characterization of stable CuS nanoparticles (CuS NPs) suitable for being used as low-cost HTMs. The colloidal dispersion can be stabilized in non-polar and halogen-free solvents that are fully compatible with the perovskite layer, extending its application to either the p-i-n or n-i-p cell configuration. In addition, such versatility is combined with a low-temperature solution based fabrication method which is fully scalable to printing role-torole (R2R) and large scale industrial processes. By using this facile approach, we present here a first demonstration of PSCs with n-i-p device configuration based on CuS NPs, which leads to short circuit currents $\left(J_{s c}\right)$ and fill factor (FF) values comparable to the state-of-theart devices $\left(J_{s c}>20 \mathrm{~mA} \cdot \mathrm{cm}^{-2} ; F F>0.7\right)$. Noteworthy, despite such optimal results, the open-circuit voltage $\left(V_{o c}\right)$ remains far below the values reported in literature $(\sim 0.8 \mathrm{~V})$, limiting the PCE to $\sim 13.5 \%$. We have further investigated the origin of such low $V_{o c}$ by analyzing the energy band alignment and charge extraction capability at the perovskite/CuS interface by using UPS and bias induced photoluminescence spectroscopy (PL). Our results demonstrate an effective hole-extraction happening at the CuS, yet followed by increased nonradiative recombination induced by a non-proper energy level alignment at the interface. Interestingly, such a behaviour could be easily avoided by using a proper interlayer to passivate the perovskite/ CuS interface, a suitable strategy that might be consider in future experiments.

\section{Experimental section}

\subsection{Synthesis of CuS nanoparticles}

In a typical procedure two separated precursor solutions were prepared. One with $0.120 \mathrm{~g}$ of sulfur powder (S, 99.9\%; Sigma Aldrich) and the other with $0.425 \mathrm{~g}$ of copper chloride dihydrate (Sigma Aldrich, $99.999 \%$ ) dissolved in $20 \mathrm{ml}$ and $10 \mathrm{ml}$ of oleylamine (Sigma Aldrich, $70 \%)$, respectively. The latter solution was heated to reach $150{ }^{\circ} \mathrm{C}$ and then sulfur solution was rapidly added. Reaction was maintained for $3 \mathrm{~min}$ and then the mixture was cooled down to room temperature. Product was collected by centrifugation at $5000 \mathrm{rpm}$ for $3 \mathrm{~min}$, washed five times with ethanol to remove oleylamine remnants and finally dispersed in toluene to obtain a $47 \mathrm{mg} / \mathrm{ml}$ stable dispersion.

\subsection{Fabrication of perovskite solar cells}

Chemically etched FTO glass (Nippon Sheet Glass) was sequentially cleaned by sonication in a $2 \%$ Helmanex soap solution, acetone and ethanol for $15 \mathrm{~min}$ each, followed by a UV-ozone treatment for $15 \mathrm{~min}$. Then a solution of $0.045 \mathrm{~g} / \mathrm{ml}$ titanium diisopropoxide bis(acetylacetonate) (Sigma-Aldrich) in anhydrous ethanol was sprayed at $450{ }^{\circ} \mathrm{C}$ to deposit a $30 \mathrm{~nm}$ thick $\mathrm{TiO}_{2}$ compact layer. To form a $200 \mathrm{~nm}$ mesoporous $\mathrm{TiO}_{2}$ layer a $0.11 \mathrm{~g} / \mathrm{ml}$ ethanol solution of a commercially available $\mathrm{TiO}_{2}$ paste (Dyesol 30NRD) was spin-coated at $2000 \mathrm{rpm}$ and substrates were annealed at $500{ }^{\circ} \mathrm{C}$ for $30 \mathrm{~min}$. Afterwards, a $0.1 \mathrm{M}$ solution of Li-TFSI in acetonitrile was deposited at $3000 \mathrm{rpm}$ for $10 \mathrm{~s}$, followed by a sintering at $500{ }^{\circ} \mathrm{C}$ for $30 \mathrm{~min}$. $\mathrm{MAPbI}_{3}$ precursor solution was prepared by mixing $1.5 \mathrm{M} \mathrm{PbI}_{2}$ and $1.5 \mathrm{M} \mathrm{CH}_{3} \mathrm{NH}_{3} \mathrm{I}$ in DMSO. CsFAMAPbIBr solution was prepared by mixing $1.15 \mathrm{M} \mathrm{PbI}_{2}, 0.19 \mathrm{M}$ $\mathrm{PbBr}_{2}, 1.1 \mathrm{M}$ formamidinium iodide (FAI) and $0.19 \mathrm{M}$ methylammonium bromide (MABr) in a mixture of DMF and DMSO with a 4:1 volume ratio (solution A). Subsequently, a solution $\mathrm{B}$ was fabricated by mixing 1.15 M CsI and 1.15 $\mathrm{M} \mathrm{PbI}_{2}$ in DMSO. Then solution A and $\mathrm{B}$ were mixed in a volume ratio of 10:1 to have the triple cation perovskite precursor. $\mathrm{MAPbI}_{3}$ was then deposited at $1000 \mathrm{rpm}$ for $10 \mathrm{~s}$ (500 rpm s${ }^{-1}$, first step) and $4000 \mathrm{rpm}$ for $30 \mathrm{~s}\left(2000 \mathrm{rpm} \mathrm{s}^{-1}\right.$, second step). $10 \mathrm{~s}$ prior to the end of the program, $100 \mu \mathrm{l}$ of chlorobenzene were dropped. For the CsFAMAPbIBr perovskite we used $2000 \mathrm{rpm}$ for

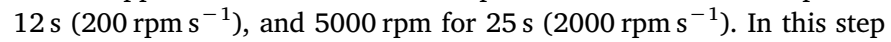
chlorobenzene was dropped $9 \mathrm{~s}$ before the end of the process. Afterwards, films were annealed at $100^{\circ} \mathrm{C}$ for $60 \mathrm{~min}$. A $47 \mathrm{mg} / \mathrm{ml} \mathrm{CuS}$ NPs dispersion was then dynamically spin-coated. Finally, the gold electrode $(70 \mathrm{~nm})$ was deposited by thermal evaporation.

\subsection{Characterization}

Transmittance of CuS NPs films were taken using PerkinElmer Lambda 1050 UV/Vis/NIR spectrophotometer. The XRD measurement was obtained with a Rigaku Ultima III X-ray diffractometer operating at $40 \mathrm{KV}$ accelerating voltage and $44 \mathrm{~mA}$ current. For Raman spectra we used a Horiba Jobin Yvon (Labram HR) equipment with a CCD detector. Cross-section SEM-images were taken in a Hitachi-4800 UHR SEM. TEM images were obtained in a Tecnai F20 Super Twin TMP equipment. The electrical characterization of the devices was performed using a $4200 S C S$ Keithley system at a voltage swept speed around $50 \mathrm{mV} / \mathrm{s}$ in combination with an Oriel sol3A sun simulator, which was calibrated to AM1.5G standard conditions using an Oriel $91,150 \mathrm{~V}$ reference cell. Conductivity measurements were performed with the four point probe employing 4200SCS Keithley. Photoelectron spectroscopy (PES) measurements were performed in a ultra-high vacuum analysis chamber 
(base pressure of $2 \times 10^{-10} \mathrm{mbar}$ ) using a He-discharge UV source (Omicron) with an excitation energy of $21.2 \mathrm{eV}$ for ultraviolet photoelectron spectroscopy (UPS) and a Al Ka X-ray source with an excitation energy of $1486.6 \mathrm{eV}$ for X-ray photoelectron spectroscopy (XPS). The photoelectron spectra were recorded using a Phoibos 100 (Specs) hemispherical energy analyzer at a pass energy of $5 \mathrm{eV}$ for the valence band, $20 \mathrm{eV}$ for the core level spectra, and $50 \mathrm{eV}$ for the survey scans. For work function determination, the secondary electron cut-off (SECO) was recorded by applying a $-10 \mathrm{~V}$ sample bias to clear the analyzer work function. A mixed Gaussian/Lorentzian peak shape and a Shirley type background were employed for XPS peak fitting with the XPS Peak 4.1 software. Samples were illuminated under white halogen lamp at a power of $150 \mathrm{~mW} \cdot \mathrm{cm}-2$ (daylight rendering spectrum).

\section{Results and discussion}

The CuS nanocrystals were prepared by using a modified version of the synthesis reported by Zhang et al. [37], in order to achieve a stable and high concentrated dispersion in non-polar solvents. In brief, we prepared two separated precursor solutions containing sulfur powder and copper chloride respectively, both dissolved in oleylamine. The latter was first heated to $150^{\circ} \mathrm{C}$ and then sulfur was rapidly added, completing the reaction after only few minutes. CuS nanoparticles were then collected from centrifugation and dispersed in toluene. The detailed crystal analysis and size-morphology are shown in Fig. 1. The Xray diffraction (XRD) pattern of the as prepared material deposited by drop casting is present in Fig. 1a. In agreement with previous works, our results demonstrate several diffraction peaks at $27.21^{\circ}, 29.36^{\circ}$, $32.35^{\circ}, 33.52^{\circ}, 48.09^{\circ}, 52.33^{\circ}$ and $59.05^{\circ}$ corresponding to the hexagonal CuS phase previously reported in literature [38]. No additional peaks related to other $\mathrm{Cu}_{2-\mathrm{x}} \mathrm{S}$ phases were further detected, suggesting a pure CuS phase with a 1:1 stoichiometry. Fig. 1b shows the size-morphology of the crystalline nanoparticles observed by electron transmission microscopy (TEM). As observed in the TEM-images, the nanocrystals exhibit a 2D disk-like shape with a maximum size of $20 \mathrm{~nm}$ and
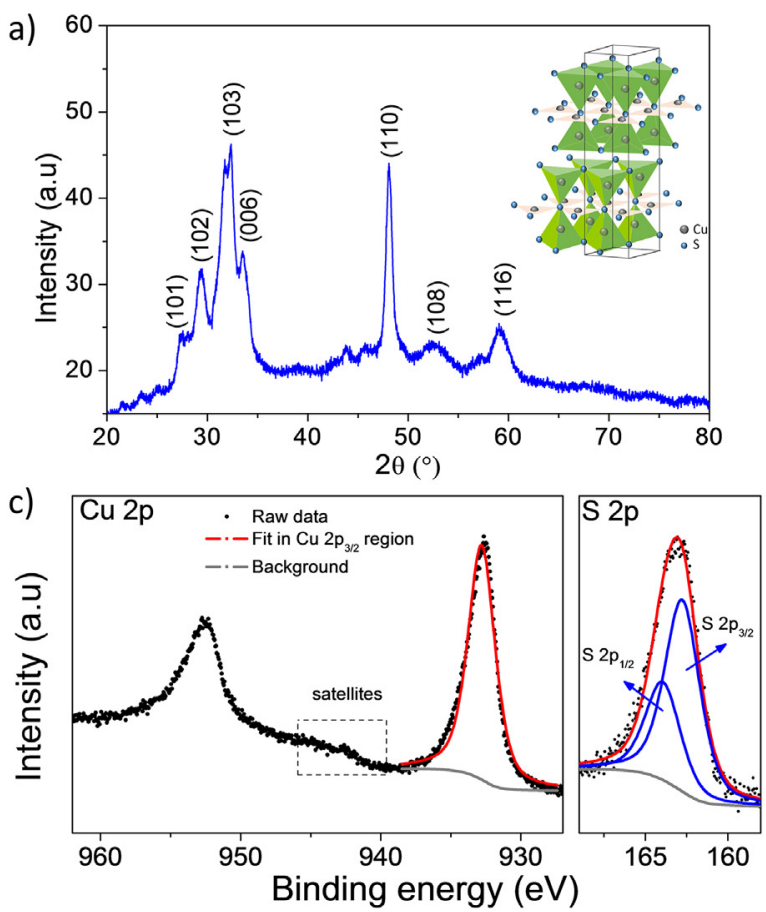

a thickness around $4 \mathrm{~nm}$, closely resembling those previously obtained through water-based synthetic strategies [37] (see also Fig. S1a and S1b). In addition, two intense Raman active phonon modes around 60 and $475 \mathrm{~cm}^{-1}$ are expected for covellite phase, which can be easily detected in the as prepared sample, shown in Fig. S1c and Fig. S1d [30]. The peaks, assigned to the stretching vibrational modes of $\mathrm{S}-\mathrm{S}$ covalent bonds [39], provide additional support to the formation of CuS.

To verify the exact chemical composition we also performed X-Ray photoelectron spectroscopy (XPS) measurements. Fig. 1c reports the core level spectrum of $\mathrm{Cu} 2 \mathrm{p}$ and $\mathrm{S} 2 \mathrm{p}$ obtained for the thin films deposited on FTO. As observed, the $\mathrm{Cu} 2 \mathrm{p}_{3 / 2}, \mathrm{~S} 2 \mathrm{p}_{1 / 2}$ and $\mathrm{S} 2 \mathrm{p}_{3 / 2}$ bands located at $932.8 \mathrm{eV}, 162.59 \mathrm{eV}$ and $163.77 \mathrm{eV}$, respectively, consist of one contribution each, hence one chemical species. However, we note here that assessing the chemical state of $\mathrm{Cu}$ can be difficult from the XPS main peak position. In particular, we highlight the presence of satellite features (between $940 \mathrm{eV}$ and $946 \mathrm{eV}$ ) and the binding energy position of the LMM Auger peak, as revealed in Fig. S2. The observation of the $\mathrm{Cu}$ shake-up satellite at ca. $943 \mathrm{eV}$, and the strong peak corresponding to the Cu LMM Auger line at ca. $568.8 \mathrm{eV}$ in the XPS survey (Fig. S2) are the signature for the presence of $\mathrm{Cu}(\mathrm{II})$, instead of $\mathrm{Cu}(\mathrm{I})$ in the material, discarding any additional mixed phase [40,41]. This is supported by the close to one Cu:S relative ratio estimated from the XPS results. Moreover, in agreement with previous works, our CuS nanodisks also exhibit an optical band gap around $2.4 \mathrm{eV}[42,43]$ and a single, intense extinction band at NIR wavelengths (>900 nm) (see Fig. 1d). Such a behaviour has been well documented in CuS nanocrystals, revealing its characteristic plasmonic-semiconductor dual nature.

To test $\mathrm{CuS}$ as a sole HTM we prepared mesoporous PSCs containing $\mathrm{MAPbI}_{3}$ and CsFAMAPbIBr as the photoactive material in order to observe device photovoltaic response and analyze interactions between $\mathrm{CuS}$ and corresponding perovskite at the interface. We employed $\mathrm{TiO}_{2}$ as electron blocking layer and CuS NPs dispersion as the HTM deposited by spin-coating technique at different rates. A statistical analysis of the photovoltaic parameters as a function of the HTM thickness is shown in

b)
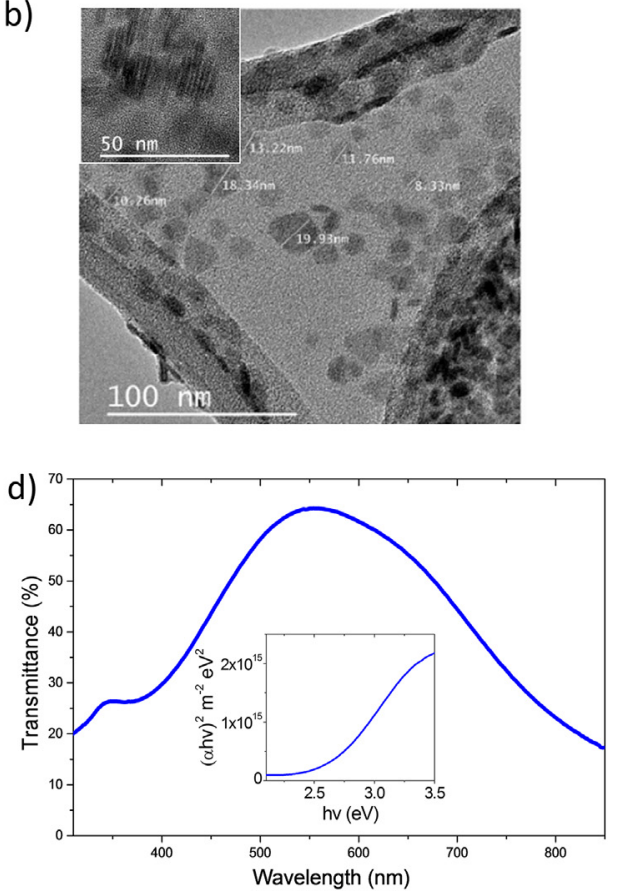

Fig. 1. Synthesized CuS characterization. (a) XRD diffractogram of dried CuS nanoparticles showing the characteristic peaks of covellite copper sulfide. Inset: scheme of crystal structure. (b) TEM image showing the flake-like shape of CuS nanoparticles. (c) XPS core level spectra of Cu 2p, and S 2p for CuS. The fitting was restricted to the part of the XP spectra corresponding to $\mathrm{Cu} 2 \mathrm{p}_{2 / 3}$ for $\mathrm{Cu} 2 \mathrm{p}$. The shake-up satellite found between $940 \mathrm{eV}$ and $946 \mathrm{eV}$ (dashed rectangle) is characteristic of $\mathrm{Cu}$ (II). (d) Transmittance spectrum of a CuS film deposited on glass and band gap estimation (inset). 

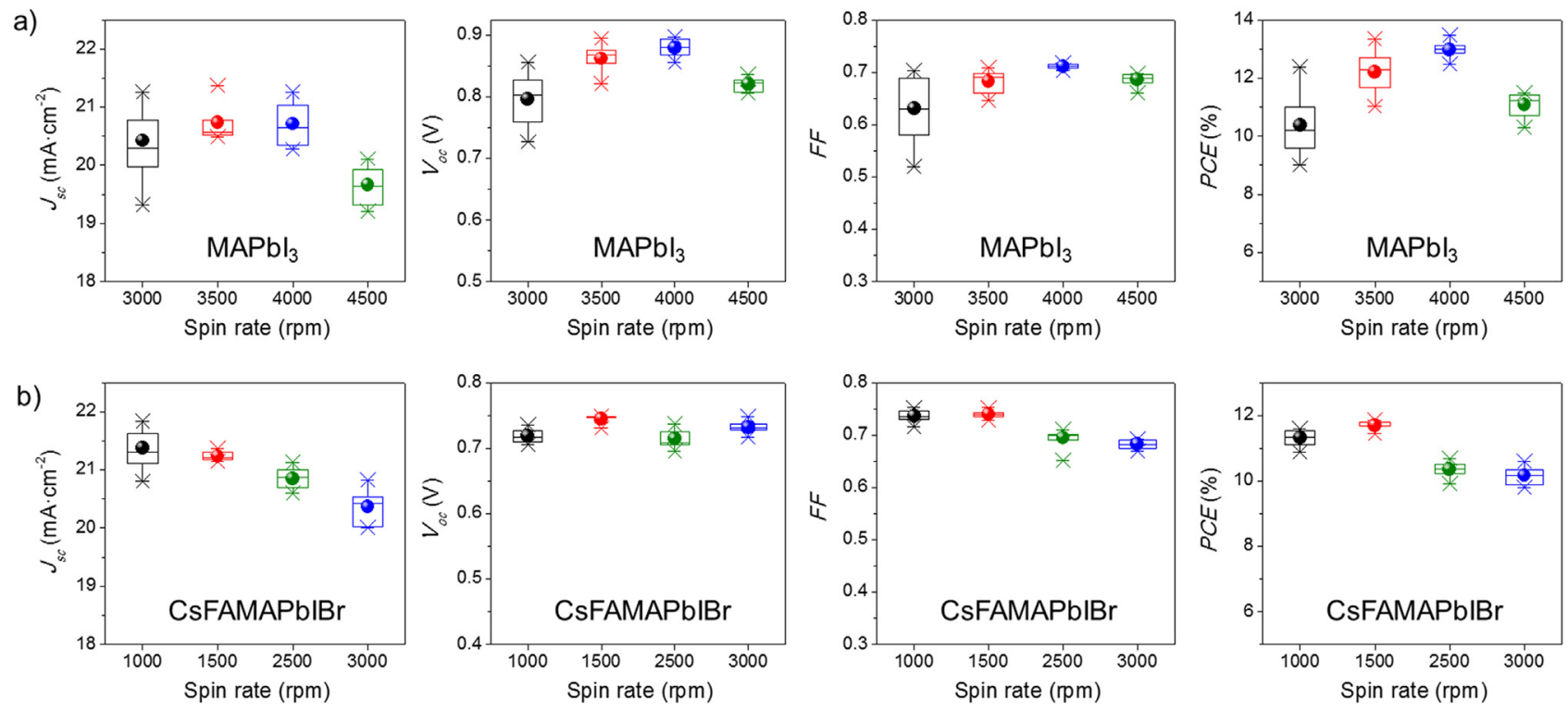

c)

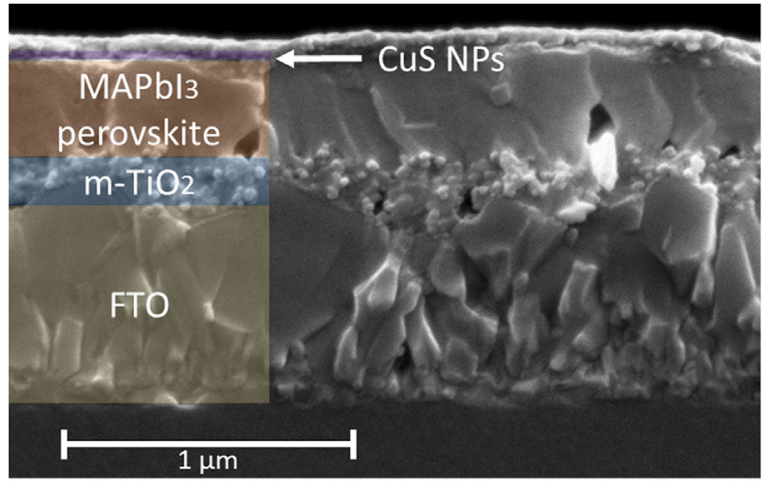

d)

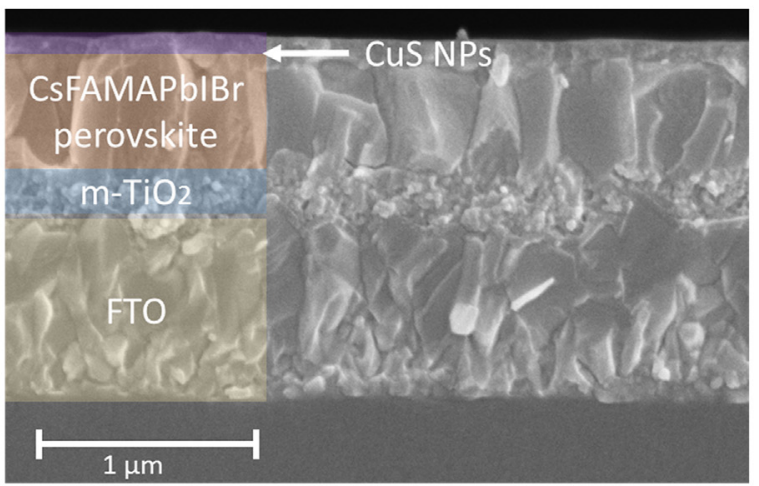

Fig. 2. Photovoltaic parameters obtained for 80 devices (10 per condition) prepared for the CuS deposition optimization on (a) MAPbI 3 and (b) CsFAMAPbIBr perovskite. Cross-section SEM images of (c) $\mathrm{MAPbI}_{3}$ and (d) CsFaMAPbIBr based PSCs.

Table 1

Photovoltaic parameters of PSCs employing CuS and Spiro-OMeTAD as HTM. The statistics were extracted from $>130$ devices (at least 10 per condition). In parenthesis the maximum PCE obtained with CuS.

\begin{tabular}{|c|c|c|c|c|}
\hline $\begin{array}{l}\text { Spinner speed } \\
(\mathrm{rpm})\end{array}$ & $\mathrm{V}_{\mathrm{oc}}$ & $\mathrm{J}_{\mathrm{sc}}$ & FF & PCE \\
\hline \multicolumn{5}{|c|}{$\mathrm{CH}_{3} \mathrm{NH}_{3} \mathrm{PbI}_{3}$ perovskite } \\
\hline Without HTM & $0.57 \pm 0.03$ & $1.07 \pm 0.28$ & $0.69 \pm 0.02$ & $0.42 \pm 0.18$ \\
\hline 3000 & $0.82 \pm 0.04$ & $20.46 \pm 0.61$ & $0.62 \pm 0.06$ & $10.45 \pm 1.25$ \\
\hline 3500 & $0.87 \pm 0.02$ & $20.92 \pm 0.31$ & $0.69 \pm 0.02$ & $12.49 \pm 0.75$ \\
\hline 4000 & $0.88 \pm 0.01$ & $20.72 \pm 0.36$ & $0.71 \pm 0.01$ & $\begin{array}{c}13.01 \pm 0.29 \\
(13.47)\end{array}$ \\
\hline 4500 & $0.83 \pm 0.01$ & $19.83 \pm 0.34$ & $0.69 \pm 0.01$ & $11.39 \pm 0.41$ \\
\hline $\begin{array}{l}\text { Spiro- } \\
\quad \text { OMeTAD }\end{array}$ & $1.04 \pm 0.03$ & $21.77 \pm 0.31$ & $0.74 \pm 0.02$ & $16.98 \pm 0.43$ \\
\hline \multicolumn{5}{|c|}{$\left(\mathrm{FAPbI}_{3}\right)_{0.78}\left(\mathrm{MAPbBr}_{3}\right)_{0.14}\left(\mathrm{CsPbI}_{3}\right)_{0.08}$ perovskite } \\
\hline Without HTM & $0.42 \pm 0.02$ & $8.16 \pm 0.19$ & $0.48 \pm 0.03$ & $1.64 \pm 0.19$ \\
\hline 1000 & $0.72 \pm 0.01$ & $21.57 \pm 0.33$ & $0.73 \pm 0.01$ & $11.37 \pm 0.24$ \\
\hline 1500 & $0.75 \pm 0.02$ & $21.23 \pm 0.07$ & $0.74 \pm 0.01$ & $\begin{array}{c}11.72 \pm 0.12 \\
(11.85)\end{array}$ \\
\hline 2000 & $0.73 \pm 0.02$ & $19.95 \pm 0.16$ & $0.71 \pm 0.02$ & $10.33 \pm 0.42$ \\
\hline 2500 & $0.71 \pm 0.01$ & $20.91 \pm 0.18$ & $0.69 \pm 0.02$ & $10.35 \pm 0.22$ \\
\hline 3000 & $0.73 \pm 0.01$ & $20.29 \pm 0.28$ & $0.68 \pm 0.01$ & $10.15 \pm 0.27$ \\
\hline $\begin{array}{l}\text { Spiro- } \\
\text { OMeTAD }\end{array}$ & $0.92 \pm 0.01$ & $21.95 \pm 0.31$ & $0.76 \pm 0.02$ & $15.27 \pm 0.23$ \\
\hline
\end{tabular}

Fig. $2 \mathrm{a}$ and $\mathrm{b}$ and Table 1, which is proportional to the rate employed during the spin-deposition. Interestingly, the optimal conditions for CuS were very different depending on the perovskite employed, probably due to the distinct surface roughness achieved for each layer $[44,45]$. In particular, we highlight the different morphology and grain-crystals observed in the cross-sectional scanning electron microscopy (SEM) images presented in Fig. $2 \mathrm{c}$ and $\mathrm{d}$, which clearly demonstrate a very smooth and compact surface for $\mathrm{MAPbI}_{3}$ compared to CsFAMAPbIBr. This allows the use of much thinner HTM layers without the presence of shunts. Therefore, only $55 \mathrm{~nm}$ CuS NPs were necessary to properly cover $\mathrm{MAPbI}_{3}$ layer, while $120 \mathrm{~nm}$ were required for CsFAMAPbIBr. As a consequence, we achieved a PCE of $\sim 13.5 \%$ for $\mathrm{MAPbI}_{3}$ and $\sim 12 \%$ for CsFAMAPbIBr (Fig. S3). For comparison, devices without HTM exhibited extremely low $J_{s c}$ and $V_{o c}$ values (PCE below $1 \%$ in all cases), confirming the positive role of $\mathrm{CuS}$ as a hole-transporting layer. In addition, forward and reverse $\mathrm{J}-\mathrm{V}$ curves of champion devices including CuS NPs and Spiro-OMeTAD as HTM are shown in Fig. S3. As expected, some hysteresis is observed for all evaluated systems. This phenomenon has been reported elsewhere for n-i-p PSC and is attributed to ion migration inside perovskite material and specially to the formation and the release of interfacial charges in both electron and hole transporting layer contacts $[46,47]$. Stability was evaluated at ambient conditions $\left(\sim 40 \% \mathrm{RH}\right.$ and $\sim 20^{\circ} \mathrm{C}$ ) being clear the strong instability of $\mathrm{MAPbI}_{3}$ to light-induced degradation [48] as shown in Fig. 3. In particular, devices containing $\mathrm{MAPbI}_{3}$ perovskite show very poor performance after $192 \mathrm{~h}$, whereas CsFAMAPbIBr cells retain almost $80 \%$ of the initial efficiency 
a)

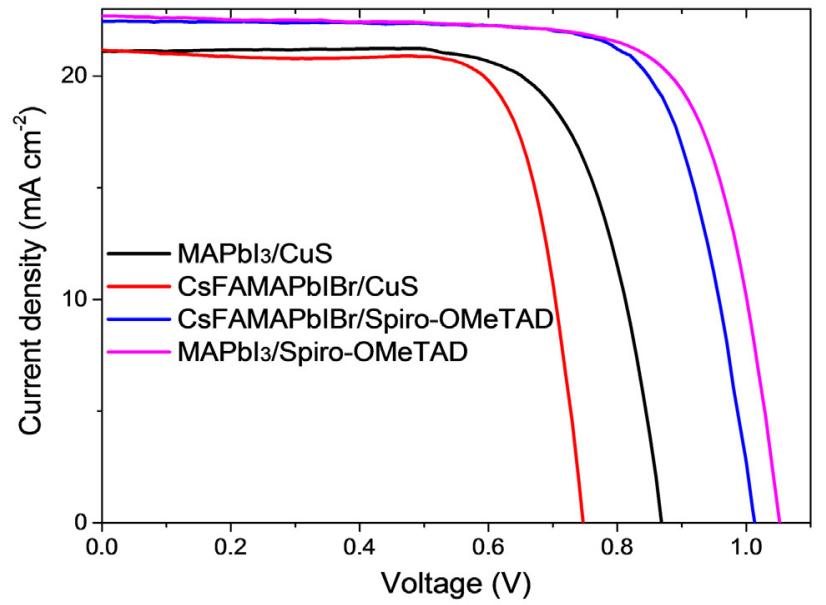

b)

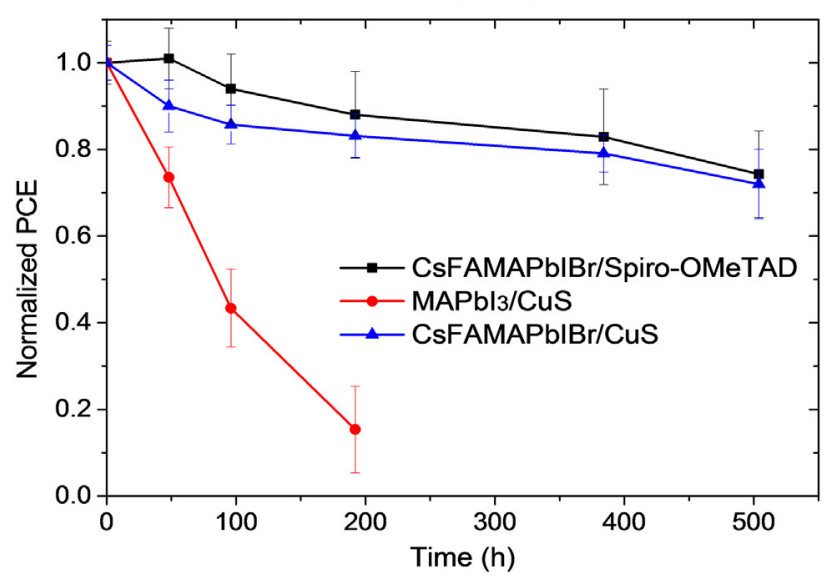

Fig. 3. (a) J-V curves of PSC employing CuS and reference Spiro-OMeTAD as HTMs in the two tested device architectures. (b) Stability test performed under 1 Sun light illumination (AM 1.5 Standard Spectrum) for PSC using CuS and Spiro-OMeTAD.

after $504 \mathrm{~h}$. The relative low efficiency loss at the latter case might be attributed to moisture permeation through CuS NPs layer due to its low thickness and considerable air humidity during the test instead of CuS intrinsic factors since different reports show the high chemical stability of the CuS nanocrystals under similar conditions [32,49]. However, according to Fig. 3b PCE retention is similar to the one observed in reference PSC employing CsFAMAPbIBr as light harvester and SpiroOMeTAD as HTM. In this system instability can be caused by SpiroOMeTAD doping agents which are known for being highly hygroscopic so that water absorption and subsequent perovskite degradation are expected [50]. As a matter of fact, it is worth underlining here that despite the modest efficiencies obtained compared with the state-of-theart, the reported results have high relevance because they imply the use of a low-temperature and solution-processed HTM which is employed for the first time solely as hole-transporting-layer avoiding the use of expensive extra electron-blocking materials. In particular, it is due to the proper precursor we have developed here which allows us to fabricate compact and high performance HTM films. Moreover, compared with Spiro-OMeTAD, the material cost at laboratory scale for the as prepared CuS-NPs is only $67.47 \mathrm{USD} / \mathrm{m}^{2}$, which is 23 times lower than Spiro-OMeTAD (1591.17 USD $/ \mathrm{m}^{2}$ ) (see cost assessment in Appendix A). In addition, CuS as HTM is compatible with friendly halogen-free solvents and printing R2R technology unlike previous reported inorganic HTMs such as CuSCN [18].

The $J-V$ curves obtained after optimization are present in Fig. 3a. Note that the results reveal excellent values for $J_{s c}$ and $F F$, closely resembling those obtained with Spiro-OMeTAD (see Table 1). There is however a remarkable deterioration for $V_{o c}$, which decreases to $0.89 \mathrm{~V}$ and $0.74 \mathrm{~V}$ for $\mathrm{MAPbI}_{3}$ and CsFAMAPbIBr respectively. Such low $V_{o c}$ values are indeed the ultimate responsible for the lower efficiencies. To further understand these observations and shed light into the origin of the low $V_{o c}$, we performed UPS measurements. Fig. 4 shows the work function $(\Phi)$ and valence band spectra for both perovskites and CuS thin films. Interestingly, the valence band spectrum of CuS (Fig. 4b) reveals some intensity that extends to the Fermi level (Fig. 4b-c). A closer inspection at that binding energy region further highlights this tailing intensity, confirming a relatively low but distinguishable density of states (DOS) at the Fermi level (inset in Fig. 4c). This provides additional support of the CuS NPs metallic character already reported in literature [51] and corroborated by conductivity measurements in Fig. S4. In addition, the valence band maxima $\mathrm{E}_{\mathrm{VBM}}$ of CsFAMAPbBrI and $\mathrm{MAPbI}_{3}$ are at $1.45 \mathrm{eV}$ and $1.21 \mathrm{eV}$ binding energy relative to $\mathrm{E}_{\mathrm{F}}$. Accordingly, both materials have a strongly n-type character, with the Fermi level pinned at their conduction band minimum (the optical band gap extracted from the absorption spectra in Fig. S5 was $1.61 \mathrm{eV}$ and $1.58 \mathrm{eV}$, respectively).

The energy level diagram deduced from the UPS measurements is

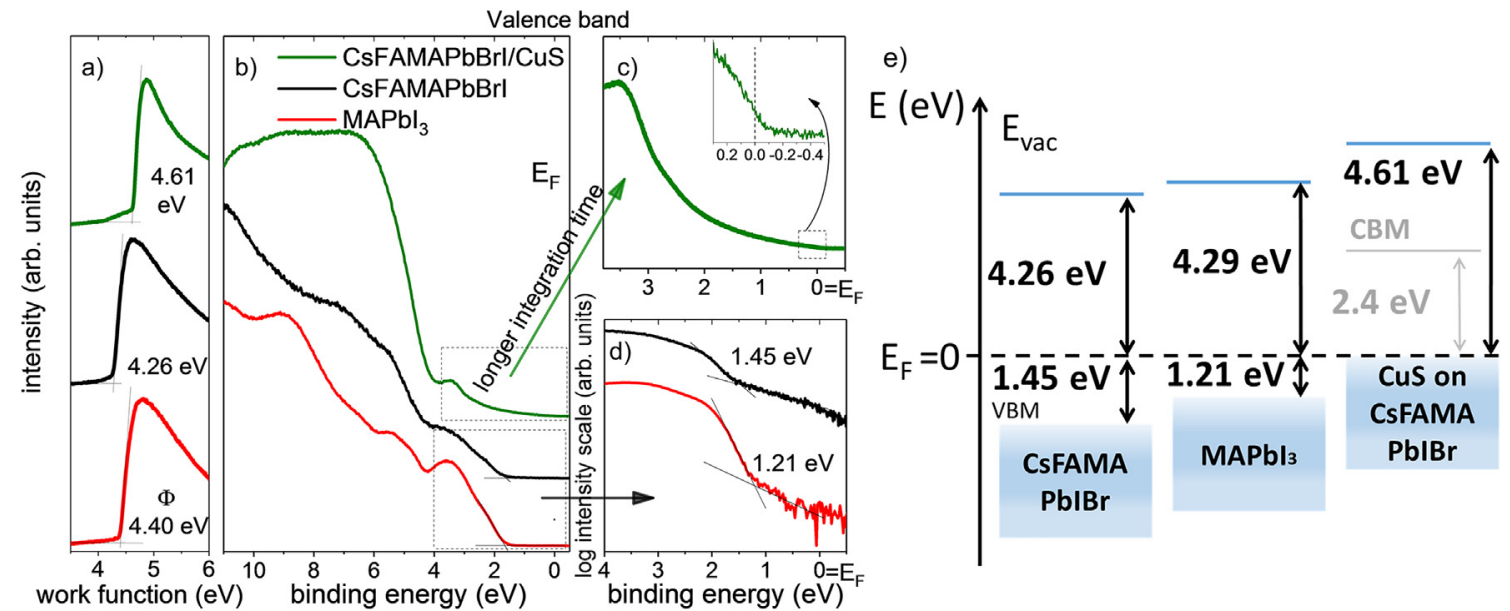

Fig. 4. UPS spectra of CsFAMAPbIBr, MAPbI3 and CuS films. (a) Secondary electron cut-offs (SECO) for work function $\phi$ determination, (b) large and short binding energy range valence band spectra of (c) CuS, (d) CsFAMAPbIBr and $\mathrm{MAPbI}_{3}$. The portion of the spectrum taken at smaller energy step in the inset in e) shows the presence of a density of states at the Fermi level for CuS. (e) Energy level diagram of CsFAMAPbIBr, $\mathrm{MAPbI}_{3}$ and CuS. The work function $\left(\phi=\mathrm{E}_{\mathrm{vac}}-\mathrm{E}_{\mathrm{F}}\right.$ ) and $\mathrm{E}_{\mathrm{VBM}}$ relative to the Fermi level $\mathrm{E}_{\mathrm{F}}$ are extracted from the UPS spectra in a) and c,d). The conduction band minimum position (CBM, in grey) is deduced from the the $2.4 \mathrm{eV}$ band gap of CuS. 


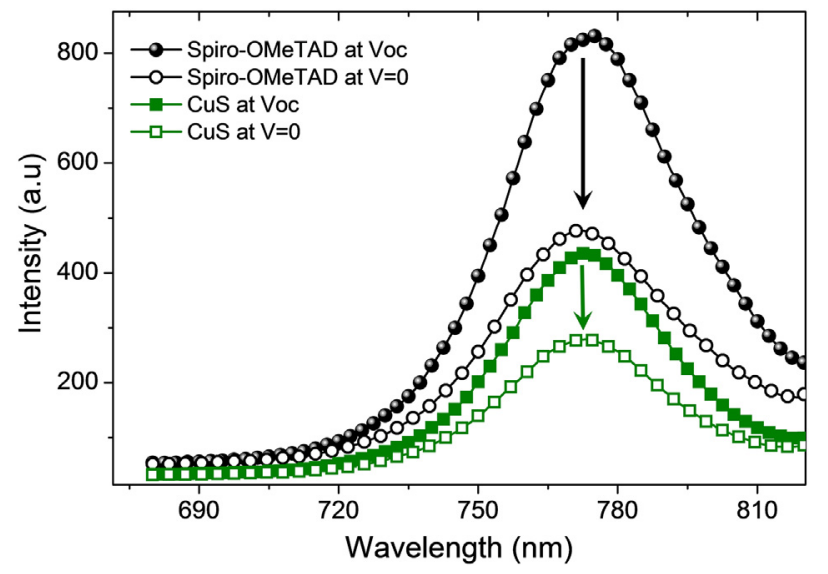

Fig. 5. Photoluminescence spectra of PSC employing Spiro-OMeTAD and CuS as HTM at $V_{o c}$ and $0 \mathrm{~V}$.

presented in Fig. 4e. The filled DOS up to the Fermi level and the position of the conduction band minimum (CBM) in grey, as deduced from the $2.4 \mathrm{eV}$ optical gap (Fig. 1d), support the suitability of CuS for hole collection and electron blocking. However, even in this case, there is a considerable energy gap $(\sim 1.2 \mathrm{eV})$ between $\mathrm{MAPbI}_{3}$ or CsFAMAPbIBr and $\mathrm{CuS} \mathrm{E}_{\mathrm{VBM}}(5.61 \mathrm{eV}, 5.71 \mathrm{eV}$ and $4.61 \mathrm{eV}$, respectively), an important energy barrier that might favor the electron-hole recombination at the interface. Interestingly, despite the really small work function difference existing between $\mathrm{TiO}_{2}$ and $\mathrm{CuS}$ contacts $(0.6 \mathrm{eV})$, we obtain a relatively high $V_{o c}\left(\sim 0.9 \mathrm{~V}\right.$ in the $\mathrm{MAPbI}_{3}$ case $)$ in fully agreement with a recent investigation published by Ravishanka et al [52].

To further understand this behaviour we evaluated the charge extraction capability of the device interface employing CsFAMAPbIBr as light harvester by comparing the photoluminescence (PL) quenching at $V_{o c}$ and short circuit conditions. Usually, the charge transfer dynamics are evaluated by comparing the PL quenching between the pristine bulk active layer and the interface behaviour. However, this gives only partial information and cannot fully explain the device mechanisms. In particular, when an HTM is present, different interface mechanisms causing PL quenching come into play, i.e. hole-transfer or distinct surface trap density. Therefore measuring the whole device under different operative conditions provides a more robust and easier analysis [53]. Because perovskites generate free carriers after photoexcitation
[54], the PL intensity will depend on whether the device is at open or short circuit conditions. In the first case, PL is proportional to the density of electron-holes which radiatively recombine. The larger is this value, the better would be the device behaviour (minor density of trap states). However, at short circuit conditions part of the charges deviates through the external path, decreasing the radiative recombination and thus, the luminescence intensity. In our case, we compared the PL quenching induced in devices containing the exact same configuration except the CuS and Spiro-OMeTAD (Fig. 5). At $V_{o c}$ conditions, the reference exhibits twice the PL as the CuS device. Given the exact same thickness of the active layer, this suggests increased intrinsic non radiative recombination for the CuS-device, which can explain the low experimental $V_{o c}$ values. At short circuiting, we also observe $\sim 20 \%$ lower quenching for the CuS-device than Spiro-OMeTAD, thus a slightly worse charge injection takes place. However, even in this case the charge transference is still happening in a considerable good extent.

Noteworthy, if we further analyze our samples by UPS upon light exposure, we observe a rigid shift of both valence band and work function by up to ca. $0.7 \mathrm{eV}$ (red curves, Fig. 6a-d), which is fully reversible once the light is removed (grey and blue curves). Such situation is equivalent to the open circuit conditions where bottom and top electrodes are not in direct electrical contact. This effect, particularly observable due to the rather metallic character of CuS (Fig. 4c), is a direct evidence of the hole-injection within CuS, which therefore behaves like an electrode with regards to the underlying perovskite. Accordingly, under illumination free charges are generated at the perovskite/CuS interface and holes are accumulated into the CuS layer, creating a space charge up to the sample surface [55]. Hence, the extent of the energy shift observed here $(0.74 \mathrm{~V})$ is directly related to the $V_{o c}$, which is further enhanced by the CuS metallic character.

\section{Conclusions}

We can therefore conclude that CuS NPs with a flake-like shape have been successfully synthesized and stabilized in non-polar solvents, fully compatible with hybrid lead halide perovskites. This material behaves for the first time as a sole p-type semiconductor in direct PSC thanks to the development of a proper colloidal precursor, which is a very cheap option to substitute expensive state-of-the art materials such as Spiro-OMeTAD (23 times lower in price). Our first observations suggest that when CuS is employed as HTM, very high values of $J_{s c}$ and FF are achieved leading to remarkable PCE close to $14 \%$. However, this
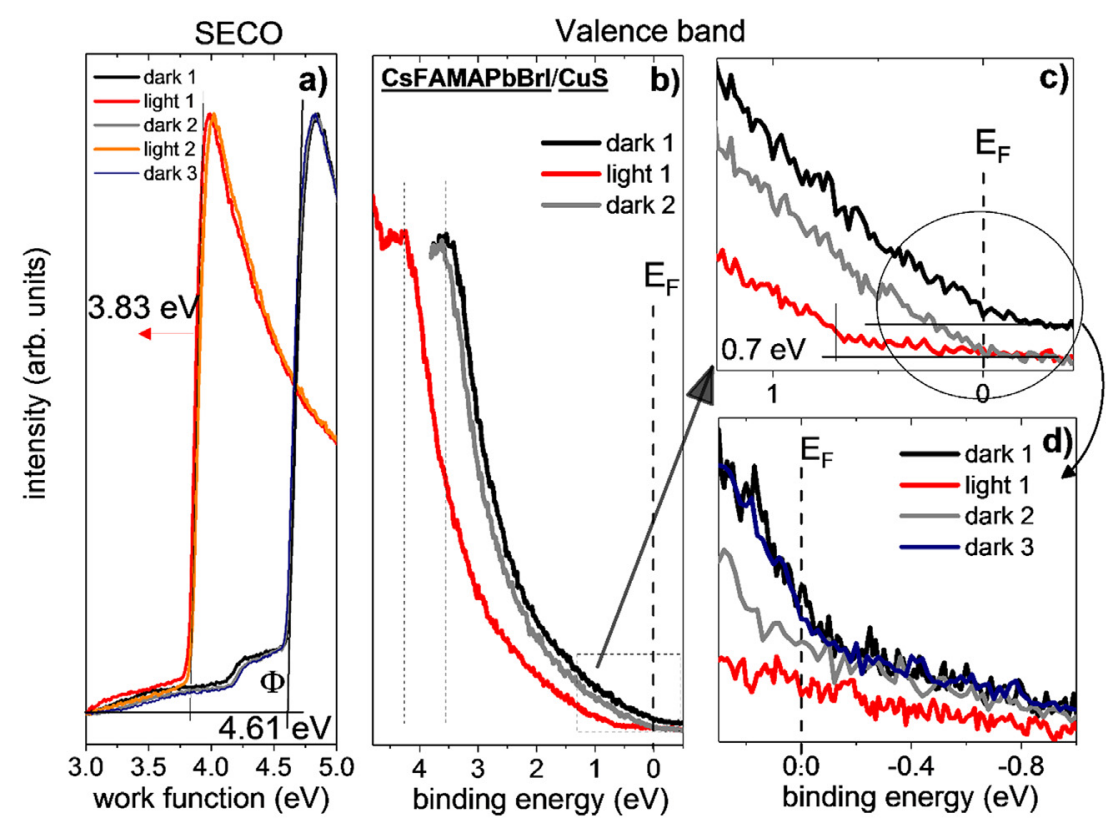

Fig. 6. UPS spectra of CuS upon white light illumination: (a) reversible SECO shift after successive measurements in dark (black), under light (red), in dark (grey), under light (orange), and in dark (blue). (b) Large binding energy range and (c) short binding energy range valence band with reversible shift upon illumination. In (d) the second measurement in dark after illumination (dark 3, blue curve) shows the complete reversibility of the light-induced shift with time. (For interpretation of the references to colour in this figure legend, the reader is referred to the web version of this article.) 
final efficiency is compromised by a significant reduced $V_{o c}$. According to the analysis we have performed here, the limited $V_{o c}$ is the result of an increased non-radiative recombination taking place at the perovskite/CuS interface, probably enhanced by the large energy barrier existing between the perovskite and CuS valence bands but at the same time $V_{o c}$ seems to be controlled by the metallic character of CuS revealed by UPS upon light exposure. Noteworthy, such a behaviour could be probably improving by using a proper interlayer to passivate the perovskite/CuS interface, different to the expensive Spiro-OMeTAD, a suitable strategy that might be considered in future experiments. These findings contribute to fill the knowledge gap about the behaviour of CuS as HTM in PSC due to the previous reported impossibility of having high performance CuS films on perovskite. Moreover, our results point out CuS as a potential extremely low-cost and stable solutionprocess alternative to the state-of-the art organic HTMs in PSC, opening the door for new improvements and applications of this material towards large-scale low-temperature produced optoelectronic devices.

\section{Author contributions}

The manuscript was written through contributions of all authors. All authors have given approval to the final version of the manuscript. C RC, M.K. N. and F. J. supervised the work.

\section{Conflict of interest}

The authors declare no competing financial interest.

\section{Acknowledgments}

We thank the Colombian "Departamento Nacional de Planeación", SGR collaborative project 2013000100184 between Empresas Públicas de Medellin, Andercol S.A., Sumicol S.A.S., and Universidad de Antioquia, for supporting this work. G.G. acknowledges the Swiss National Science Foundation (SNSF) funding through the Ambizione Energy project HYPER (grant number PZENP2_173641). We thank Borun New Material Technology for providing high quality SpiroOMeTAD.

\section{Appendix A. Supplementary data}

Supplementary data to this article can be found online at https:// doi.org/10.1016/j.apsusc.2019.01.289.

\section{References}

[1] A. Kojima, K. Teshima, Y. Shirai, T. Miyasaka, Organometal halide perovskites as visible-light sensitizers for photovoltaic cells, J. Am. Chem. Soc. 131 (2009) 6050-6051, https://doi.org/10.1021/ja809598r.

[2] W.S. Yang, B.-W. Park, E.H. Jung, N.J. Jeon, Y.C. Kim, D.U. Lee, S.S. Shin, J. Seo, E.K. Kim, J.H. Noh, S. Il Seok, Iodide management in formamidinium-lead-halide-based perovskite layers for efficient solar cells, Science 80 (356) (2017) 1376-1379, https://doi.org/10.1126/science.aan2301.

[3] J.-P. Correa-Baena, A. Abate, M. Saliba, W. Tress, T. Jesper Jacobsson, M. Gratzel, A. Hagfeldt, The rapid evolution of highly efficient perovskite solar cells, Energy Environ. Sci. 10 (2017) 710-727, https://doi.org/10.1039/C6EE03397K.

[4] H.J. Snaith, Perovskites: the emergence of a new era for low-cost, high-efficiency solar cells, J. Phys. Chem. Lett. 4 (2013) 3623-3630, https://doi.org/10.1021/ jz4020162.

[5] W. Chen, Y. Wu, Y. Yue, J. Liu, W. Zhang, X. Yang, H. Chen, E. Bi, I. Ashraful, M. Grätzel, L. Han, Efficient and stable large-area perovskite solar cells with inorganic charge extraction layers, Science 80 (350) (2015) 944-948, https://doi. org/10.1126/science.aad1015.

[6] A.T. Murray, J.M. Frost, C.H. Hendon, C.D. Molloy, D.R. Carbery, A. Walsh, Modular design of SPIRO-OMeTAD analogues as hole transport materials in solar cells, Chem. Commun. 51 (2015) 8935-8938, https://doi.org/10.1039/ c5cc02129d.

[7] C. Giansante, R. Mastria, G. Lerario, L. Moretti, I. Kriegel, F. Scotognella, G. Lanzani, S. Carallo, M. Esposito, M. Biasiucci, A. Rizzo, G. Gigli, Molecular-level switching of polymer/nanocrystal non-covalent interactions and application in hybrid solar cells, Adv. Funct. Mater. 25 (2015) 111-119, https://doi.org/10.1002/ adfm. 201401841.

[8] A. Krishna, D. Sabba, H. Li, J. Yin, P.P. Boix, C. Soci, S.G. Mhaisalkar, A.C. Grimsdale, Novel hole transporting materials based on triptycene core for high efficiency mesoscopic perovskite solar cells, Chem. Sci. 5 (2014) 2702-2709, https://doi.org/10.1039/C4SC00814F.

[9] S. Kazim, F.J. Ramos, P. Gao, M.K. Nazeeruddin, M. Grätzel, S. Ahmad, A dopant free linear acene derivative as a hole transport material for perovskite pigmented solar cells, Energy Environ. Sci. 8 (2015) 1816-1823, https://doi.org/10.1039/ c5ee00599j.

[10] G.-W. Kim, J. Kim, G.-Y. Lee, G. Kang, J. Lee, T. Park, A strategy to design a donor$\pi$-acceptor polymeric hole conductor for an efficient perovskite solar cell, Adv. Energy Mater. 5 (2015) 1500471-n/a, https://doi.org/10.1002/aenm.201500471.

[11] J.C. Brauer, Y.H. Lee, M.K. Nazeeruddin, N. Banerji, Charge transfer dynamics from organometal halide perovskite to polymeric hole transport materials in hybrid solar cells, J. Phys. Chem. Lett. (2015) 3675-3681, https://doi.org/10.1021/acs.jpclett. 5b01698.

[12] C.-Y. Chang, Y.-C. Chang, W.-K. Huang, W.-C. Liao, H. Wang, C. Yeh, B.-C. Tsai, Y.C. Huang, C.-S. Tsao, Achieving high efficiency and improved stability in large-area ITO-free perovskite solar cells with thiol-functionalized self-assembled monolayers, J. Mater. Chem. A 4 (2016) 7903-7913, https://doi.org/10.1039/C6TA02581A.

[13] M. Cai, Y. Wu, H. Chen, X. Yang, Y. Qiang, L. Han, Cost-performance analysis of perovskite solar modules, Adv. Sci. 4 (2017) 1600269, https://doi.org/10.1002/ advs.201600269.

[14] Y. W., Z. X., J. L., X. W., Y. L., C. L., S. Z., H. Z., Towards printed perovskite solar cells with cuprous oxide hole transporting layers: a theoretical design, Semicond. Sci. Technol. 30 (2015) 54004, https://doi.org/10.1088/0268-1242/30/5/054004.

[15] K. Rakstys, S. Paek, P. Gao, P. Gratia, T. Marszalek, G. Grancini, K.T. Cho, K. Genevicius, V. Jankauskas, W. Pisula, M.K. Nazeeruddin, Molecular engineering of face-on oriented dopant-free hole transporting material for perovskite solar cells with 19\% PCE, J. Mater. Chem. A 5 (2017) 7811-7815, https://doi.org/10.1039/ C7TA01718A.

[16] M.-H. Li, P.-S. Shen, K.-C. Wang, T.-F. Guo, P. Chen, Inorganic p-type contact materials for perovskite-based solar cells, J. Mater. Chem. A 3 (2015) 9011-9019, https://doi.org/10.1039/C4TA06425A.

[17] N.-G. Park, Perovskite solar cells: an emerging photovoltaic technology, Mater. Today 18 (2015) 65-72, https://doi.org/10.1016/j.mattod.2014.07.007.

[18] P. Qin, S. Tanaka, S. Ito, N. Tetreault, K. Manabe, H. Nishino, M.K. Nazeeruddin, M. Grätzel, Inorganic hole conductor-based lead halide perovskite solar cells with 12.4\% conversion efficiency, Nat Commun. 5 (2014), https://doi.org/10.1038/ ncomms 4834 .

[19] Z. Liu, A. Zhu, F. Cai, L. Tao, Y. Zhou, Z. Zhao, Q. Chen, Y.-B. Cheng, H. Zhou, Nickel oxide nanoparticles for efficient hole transport in p-i-n and n-i-p perovskite solar cells, J. Mater. Chem. A 5 (2017) 6597-6605, https://doi.org/10.1039/ C7TA01593C.

[20] M.I. Hossain, F.H. Alharbi, N. Tabet, Copper oxide as inorganic hole transport material for lead halide perovskite based solar cells, Sol. Energy 120 (2015) 370-380, https://doi.org/10.1016/j.solener.2015.07.040.

[21] W. Li, H. Dong, X. Guo, N. Li, J. Li, G. Niu, L. Wang, Graphene oxide as dual functional interface modifier for improving wettability and retarding recombination in hybrid perovskite solar cells, J. Mater. Chem. A 2 (2014) 20105-20111, https:// doi.org/10.1039/C4TA05196C.

[22] S.N. Habisreutinger, T. Leijtens, G.E. Eperon, S.D. Stranks, R.J. Nicholas, H.J. Snaith, Carbon nanotube/polymer composites as a highly stable hole collection layer in perovskite solar cells, Nano Lett. 14 (2014) 5561-5568, https://doi.org/10. 1021/nl501982b.

[23] L. Xu, L.-L. Deng, J. Cao, X. Wang, W.-Y. Chen, Z. Jiang, Solution-processed cu(in, $\mathrm{Ga})(\mathrm{S}, \mathrm{se}) 2$ nanocrystal as inorganic hole-transporting material for efficient and stable perovskite solar cells, Nanoscale Res. Lett. 12 (2017) 159, https://doi.org/ 10.1186/s11671-017-1933-z.

[24] A.J. Huckaba, P. Sanghyun, G. Grancini, E. Bastola, C.K. Taek, L. Younghui, K.P. Bhandari, C. Ballif, R.J. Ellingson, M.K. Nazeeruddin, Exceedingly cheap perovskite solar cells using Iron pyrite hole transport materials, ChemistrySelect. 1 (2016) 5316-5319, https://doi.org/10.1002/slct.201601378.

[25] H. Lei, G. Yang, X. Zheng, Z.-G. Zhang, C. Chen, J. Ma, Y. Guo, Z Chen, P. Qin, Y. Li, G. Fang, Incorporation of High-Mobility and Room-Temperature-Deposited CuxS as a Hole Transport Layer for Efficient and Stable Organo-Lead Halide Perovskite Solar Cells, Phys. Status Solidi (RRL) (2017) 1700038-n/a, https://doi.org/10.1002/solr. 201700038.

[26] D. Han, C. Wu, Q. Zhang, S. Wei, X. Qi, Y. Zhao, Y. Chen, Y. Chen, L. Xiao, Z. Zhao, Solution-processed Cu9S5 as hole transport layer for efficient and stable perovskite solar cells, ACS Appl. Mater. Interfaces (2018), https://doi.org/10.1021/acsami. 8b08888.

[27] V.E. Madhavan, I. Zimmermann, C. Roldán-Carmona, G. Grancini, M. Buffiere, A. Belaidi, M.K. Nazeeruddin, Copper thiocyanate inorganic hole-transporting material for high-efficiency perovskite solar cells, ACS Energy Lett. 1 (2016) 1112-1117, https://doi.org/10.1021/acsenergylett.6b00501.

[28] N. Arora, M.I. Dar, A. Hinderhofer, N. Pellet, F. Schreiber, S.M. Zakeeruddin, M. Grätzel, Perovskite solar cells with CuSCN hole extraction layers yield stabilized efficiencies greater than $20 \%$, Science 80 (2017) (2017), https://doi.org/10.1126/ science.aam5655.

[29] W. Ke, G. Fang, H. Lei, P. Qin, H. Tao, W. Zeng, J. Wang, X. Zhao, An efficient and transparent copper sulfide nanosheet film counter electrode for bifacial quantum dot-sensitized solar cells, J. Power Sources 248 (2014) 809-815, https://doi.org/ 10.1016/j.jpowsour.2013.10.028.

[30] K.-J. Huang, J.-Z. Zhang, Y. Fan, One-step solvothermal synthesis of different morphologies CuS nanosheets compared as supercapacitor electrode materials, J. 
Alloys Compd. 625 (2015) 158-163, https://doi.org/10.1016/j.jallcom.2014.11. 137.

[31] H. Nozaki, K. Shibata, N. Ohhashi, Metallic hole conduction in CuS, J. Solid State Chem. 91 (1991) 306-311, https://doi.org/10.1016/0022-4596(91)90085-V.

[32] H. Rao, W. Sun, S. Ye, W. Yan, Y. Li, H. Peng, Z. Liu, Z. Bian, C. Huang, Solutionprocessed CuS NPs as an inorganic hole-selective contact material for inverted planar perovskite solar cells, ACS Appl. Mater. Interfaces 8 (2016) 7800-7805, https://doi.org/10.1021/acsami.5b12776.

[33] X. Jiang, Y. Xie, J. Lu, W. He, L. Zhu, Y. Qian, Preparation and phase transformation of nanocrystalline copper sulfides (Cu9S8, Cu7S4 and CuS) at low temperature, J. Mater. Chem. 10 (2000) 2193-2196, https://doi.org/10.1039/B0024860.

[34] H. Grijalva, M. Inoue, S. Boggavarapu, P. Calvert, Amorphous and crystalline copper sulfides, CuS, J. Mater. Chem. 6 (1996) 1157-1160, https://doi.org/10. 1039/JM9960601157.

[35] J. Tirado, D. Ramirez, R. Betancur, F. Jaramillo, Low-cost semi-transparent copper sulfide electrode for indium-tin-oxide-free perovskite solar cells, Thin Solid Films 662 (2018) 90-96, https://doi.org/10.1016/j.tsf.2018.07.037.

[36] P. Waldner, W. Sitte, Digenite Cu2 - x S: thermodynamic analysis of sulfur activities, Chem. Mon 143 (2012) 1215-1218, https://doi.org/10.1007/s00706-0120751-3.

[37] H. Zhang, Y. Zhang, J. Yu, D. Yang, Phase-selective synthesis and self-assembly of monodisperse copper sulfide nanocrystals, J. Phys. Chem. C 112 (2008) 13390-13394, https://doi.org/10.1021/jp801507h.

[38] X. Chen, Z. Wang, X. Wang, R. Zhang, X. Liu, W. Lin, Y. Qian, Synthesis of novel copper sulfide hollow spheres generated from copper (II)-thiourea complex, J. Cryst. Growth 263 (2004) 570-574, https://doi.org/10.1016/j.jcrysgro.2003.12. 004.

[39] N.A. Yeryukov, A.G. Milekhin, L.L. Sveshnikova, T.A. Duda, L.D. Pokrovsky, A.K. Gutakovskii, S.A. Batsanov, E.E. Rodyakina, A.V. Latyshev, D.R.T. Zahn, Synthesis and characterization of CuxS $(\mathrm{x}=1-2)$ nanocrystals formed by the Langmuir-Blodgett technique, J. Phys. Chem. C 118 (2014) 23409-23414, https:// doi.org/10.1021/jp507355t.

[40] Advanced analysis of copper X-ray photoelectron spectra, J. Phys. Chem. C 49 (2017) 1325-1334, https://doi.org/10.1002/sia.6239.

[41] D.L. Perry, J.A. Taylor, X-ray photoelectron and Auger spectroscopic studies of $\mathrm{Cu}_{2} \mathrm{~S}$ and CuS, J. Mater. Sci. Lett. 5 (1986) 384-386, https://doi.org/10.1007/ BF01672333.

[42] J. Li, T. Jiu, G.-H. Tao, G. Wang, C. Sun, P. Li, J. Fang, L. He, Manipulating surface ligands of copper sulfide nanocrystals: synthesis, characterization, and application to organic solar cells, J. Colloid Interface Sci. 419 (2014) 142-147, https://doi.org/ 10.1016/j.jcis.2013.12.057.

[43] M. Adelifard, H. Eshghi, M.M.B. Mohagheghi, An investigation on substrate temperature and copper to sulphur molar ratios on optical and electrical properties of nanostructural CuS thin films prepared by spray pyrolysis method, Appl. Surf. Sci. 258 (2012) 5733-5738, https://doi.org/10.1016/j.apsusc.2012.02.079.

[44] J. Ye, X. Zhang, L. Zhu, H. Zheng, G. Liu, H. Wang, T. Hayat, X. Pan, S. Dai,
Enhanced morphology and stability of high-performance perovskite solar cells with ultra-smooth surface and high fill factor via crystal growth engineering, Sustain. Energy Fuels. 1 (2017) 907-914, https://doi.org/10.1039/C7SE00036G.

[45] Z. Yao, W. Wang, H. Shen, Y. Zhang, Q. Luo, X. Yin, X. Dai, J. Li, H. Lin, CH3NH3PbI3 grain growth and interfacial properties in meso-structured perovskite solar cells fabricated by two-step deposition, Sci. Technol. Adv. Mater. 18 (2017) 253-262, https://doi.org/10.1080/14686996.2017.1298974.

[46] B. Chen, M. Yang, S. Priya, K. Zhu, Origin of J-V hysteresis in perovskite solar cells, J. Phys. Chem. Lett. 7 (2016) 905-917, https://doi.org/10.1021/acs.jpclett. $6 \mathrm{~b} 00215$.

[47] S.A.L. Weber, I.M. Hermes, S.-H. Turren-Cruz, C. Gort, V.W. Bergmann, L. Gilson, A. Hagfeldt, M. Graetzel, W. Tress, R. Berger, How the formation of interfacial charge causes hysteresis in perovskite solar cells, Energy Environ. Sci. 11 (2018) 2404-2413, https://doi.org/10.1039/C8EE01447G.

[48] M. Saliba, T. Matsui, J.-Y. Seo, K. Domanski, J.-P. Correa-Baena, M.K. Nazeeruddin, S.M. Zakeeruddin, W. Tress, A. Abate, A. Hagfeldt, M. Gratzel, Cesium-containing triple cation perovskite solar cells: improved stability, reproducibility and high efficiency, Energy Environ. Sci. 9 (2016) 1989-1997, https://doi.org/10.1039/ C5EE03874J.

[49] M. Ye, X. Wen, N. Zhang, W. Guo, X. Liu, C. Lin, In situ growth of CuS and Cu1.8S nanosheet arrays as efficient counter electrodes for quantum dot-sensitized solar cells, J. Mater. Chem. A 3 (2015) 9595-9600 https://doi.org/10.1039/ C5TA00390C.

[50] Q. Luo, Y. Zhang, C. Liu, J. Li, N. Wang, H. Lin, Iodide-reduced graphene oxide with dopant-free spiro-OMeTAD for ambient stable and high-efficiency perovskite solar cells, J. Mater. Chem. A 3 (2015) 15996-16004, https://doi.org/10.1039/ C5TA02710A.

[51] X. Xu, J. Bullock, L.T. Schelhas, E.Z. Stutz, J.J. Fonseca, M. Hettick, V.L. Pool, K.F. Tai, M.F. Toney, X. Fang, A. Javey, L.H. Wong, J.W. Ager, Chemical bath deposition of p-type transparent, highly conducting (CuS)x:(ZnS)1-x nanocomposite thin films and fabrication of Si heterojunction solar cells, Nano Lett. 16 (2016) 1925-1932, https://doi.org/10.1021/acs.nanolett.5b05124.

[52] S. Ravishankar, S. Gharibzadeh, C. Roldán-Carmona, G. Grancini, Y. Lee, M. Ralaiarisoa, A.M. Asiri, N. Koch, J. Bisquert, M.K. Nazeeruddin, Influence of charge transport layers on open-circuit voltage and hysteresis in perovskite solar cells, Joule 2 (2018) 788-798, https://doi.org/10.1016/j.joule.2018.02.013.

[53] K. Tvingstedt, O. Malinkiewicz, A. Baumann, C. Deibel, H.J. Snaith, V. Dyakonov, H.J. Bolink, Radiative efficiency of lead iodide based perovskite solar cells, Sci. Rep. 4 (2014) 6071, https://doi.org/10.1038/srep06071.

[54] V. D'Innocenzo, G. Grancini, M.J.P. Alcocer, A.R.S. Kandada, S.D. Stranks, M.M. Lee, G. Lanzani, H.J. Snaith, A. Petrozza, Excitons versus free charges in organo-lead tri-halide perovskites, Nat. Commun. 5 (2014) 3586, https://doi.org/10. 1038/ncomms4586.

[55] J. Frisch, M. Schubert, E. Preis, J.P. Rabe, D. Neher, U. Scherf, N. Koch, Full electronic structure across a polymer heterojunction solar cell, J. Mater. Chem. 22 (2012) 4418-4424, https://doi.org/10.1039/C1JM14968G. 